\title{
Dissecting respiratory disease heterogeneity through the genetics of diffusing capacity
}

\author{
Brian D. Hobbs (1) and Michael H. Cho \\ Affiliation: Channing Division of Network Medicine and Division of Pulmonary and Critical Care Medicine, \\ Brigham and Women's Hospital, Boston, MA, USA. \\ Correspondence: Michael H. Cho, Channing Division of Network Medicine, 181 Longwood Ave, \#451, Boston, \\ MA 02115, USA. E-mail: remhc@achanning.harvard.edu
}

@ERSpublications

Genome-wide association studies of physiological measurements, such as diffusing capacity, can help to explore the genetic underpinnings of respiratory disease clinical heterogeneity http://ow.ly/aXMx $30 \mathrm{ltt} 4 \mathrm{f}$

Cite this article as: Hobbs $\mathrm{BD}$, Cho $\mathrm{MH}$. Dissecting respiratory disease heterogeneity through the genetics of diffusing capacity. Eur Respir J 2018; 52: 1801468 [https://doi.org/10.1183/13993003.01468-2018].

Genome-wide association studies (GWASs) have allowed the robust and replicable identification of novel genomic regions associated with respiratory diseases. For instance, in chronic obstructive pulmonary disease (COPD), nearly all of the described genetic risk regions were not previously known to play a role in COPD pathogenesis [1-9]. However, where sample size is critical to discovery of new genetic risk regions in GWASs, large GWASs of lung function (and lung function extremes) in the general population have made great strides in describing the genetic risk regions contributing to the observed population variability in spirometry, and in risk of COPD [10-16]. Interestingly, the genetic risk regions contributing to the observed population variability of forced expiratory volume in $1 \mathrm{~s}$ (FEV1) and FEV1 to forced vital capacity (FVC) ratio can be aggregated into genetic risk scores which are predictive of COPD [14, 16, 17].

Despite the wealth of information GWASs of COPD and lung function have provided toward understanding the genetic architecture of COPD, these studies have only marginally addressed the observed clinical and physiological heterogeneity of COPD. Machine learning techniques are one approach to discover enrichment of association of known genetic risk loci with a subgroup of COPD patients with shared clinical features [18]. The differential strength of association of COPD risk variants with COPD-related phenotypes, such as quantitative imaging features, can also be used to explore the genetic basis for COPD heterogeneity $[9,19]$. Aside from these techniques, studying the genetics of sub-phenotypes and key physiological features of respiratory diseases may allow us to more directly evaluate the biological processes contributing to the clinical and physiological heterogeneity of these diseases, and in particular, COPD. GWASs of quantitative computed tomography emphysema [20, 21], despite smaller sample sizes and reduced statistical power, have demonstrated that using a more biologically specific phenotype allows discovery of variants not previously discovered in COPD GWASs, including the genome-wide significant identification of variants correlated with the first-described COPD genetic susceptibility variant, the $\mathrm{Z}$ allele in SERPINA1. Genetic studies of COPD-related clinical and physiologic features including emphysema pattern [22], emphysema distribution [23], resting oxygenation [24], pulmonary artery enlargement [25], chronic bronchitis [26] and the asthma/COPD overlap syndrome [27], have highlighted a genetic basis for the observed clinical heterogeneity of COPD and invite ongoing work into further describing the genetic susceptibility to specific features of COPD and other respiratory diseases. 
In this issue of the European Respiratory Journal, TERZIKHAn et al. [28] present a heritability analysis and GWAS of the diffusing capacity of the lung measured by carbon monoxide uptake (DLCO) as well as the transfer coefficient of the lung for carbon monoxide (DLCO/VA or KCO). DLCO and DLCO/VA are physiological traits impaired in the advanced stages of a number of complex respiratory diseases including COPD, interstitial lung disease and pulmonary hypertension. The heritability (the proportion of phenotypic variance explained by genetics) of DLCO was previously described in adult twins from the Swedish Twin Registry [29]; however, this is the first study examining the genome-wide genetic variant associations with either DLCO or DLCO/VA. In a meta-analysis of 8372 individuals from two European-ancestry studies, the Framingham Heart Study and the Rotterdam Study, the authors identified a genome-wide association of an uncommon variant (rs17280293, gnomAD European MAF 2.8\%) in the ADGRG6 (formerly known as GPR126) locus with DLCO/VA.

The association of rs 17280293 with DLCO/VA is given additional credence since variants close to ADGRG6 have previously been associated with FEV1/FVC ratio $[10,12-14]$, COPD [9] and various anthropomorphic measurements in large GWASs. Notably, recent lung function GWASs have identified two independent loci at $A D G R G 6$ associated with $\mathrm{FEV} 1 / \mathrm{FVC}$ ratio $[13,14]$, where the lead variant at the second ADGRG6 locus is in linkage disequilibrium $\left(\mathrm{R}^{2}=0.93\right.$ in Europeans) with rs 17280293 , which is associated with DLCO/VA in this journal. TERZIKHAN et al. [28] performed fine mapping and reported a 0.72 posterior probability for rs17280293 to explain the observed DLCO/VA association. Further, the authors report that rs17280293 is a missense variant predicted to be deleterious to ADGRG6 protein structure in two different functional impact prediction tools. In a separate lung tissue dataset, the authors reported significantly lower levels of ADGRG6 in lung tissue in association with both COPD case status and lower DLCO/VA. These data suggest rs17280293 is the causal variant and ADGRG6 is the causal gene for the $D \mathrm{LCO} / \mathrm{VA}$ association at this locus. ADGRG6 is plausible effector gene, with a role in angiogenesis. Despite this compelling evidence, a more definitive link between rs17280293 and expression or function of ADGRG6 will need to be established.

The ADGRG6 locus is complex. In addition to being previously described in association with lung function and COPD, variants at this locus are also associated with height, and previous studies have described two independent associations at this locus. Terziknan et al. [28] assessed for possible confounding of the ADGRG6 locus DLCO/VA association by adjusting for FEV1/FVC ratio and showed unchanged effect of rs17280293 on DLCO/VA. The association was further shown to be robust to adjustment for height as well as quantitative emphysema in a subset of individuals. These data suggest that the ADGRG6 locus identified by the authors is independently associated with $D \mathrm{LCO} / V \mathrm{~A}$ level when considering FEV1/FVC ratio, height, or amount of emphysema. However, DLCO is a complex phenotype, affected not just by emphysema but by pulmonary vasculature and haemoglobin; $D \mathrm{LCO} / \mathrm{VA}$ is further influenced by body size but does not scale monotonically with lung volume [30, 31]. The sensitivity analyses performed by the authors are subject to power considerations and further work (for example, using larger sample sizes with more detailed phenotyping and mediation analysis) will need to be done to disentangle relationship of traits at the ADGRG6 locus marked by rs17280293 to determine if traits are truly independent in their association to the ADGRG6 locus or if the associations are explained by un-modelled mediation of the traits by each other.

The work by TERziKhan et al. [28] in this issue of the European Respiratory Journal illustrates how the genetic study of more discrete physiologic traits, such as DLCO and DLCO/VA, impaired at the advanced stages of complex respiratory disease, can contribute to our understanding of the genetic basis for the clinical heterogeneity of respiratory diseases. Additionally, this study demonstrates the importance of sample size for genetic discovery and replication, where only a single loci genome-wide significant association with DLCO/VA (and not DLCO) was described and has yet to be replicated. Despite careful consideration of the explanatory model for the relationship of rs17280293 to DLCO/VA levels, this work highlights the difficult task left by genetic discovery, where even genetic associations with high statistical confidence mark the beginning of our journey to biologically cohesive functional descriptions of genetic loci, let alone translation to clinical relevance. In summary, TerzikHAN et al. [28] have contributed valuable information to our understanding of complex respiratory diseases and we look forward to future larger investigation of the genetic associations and functional follow-up of DLCO and DLCO/VA.

Conflict of interest: B.D. Hobbs has nothing to disclose. M.H. Cho reports grant funding from GSK, outside the submitted work.

Support statement: This work was funded by the National Institutes of Health, National Heart, Lung, and Blood Institute (K08 HL136928, R01 HL113264, R01 HL135142, R01 HL137927). Funding information for this article has been deposited with the Crossref Funder Registry. 


\section{References}

1 Pillai SG, Ge D, Zhu G, et al. A genome-wide association study in chronic obstructive pulmonary disease (COPD): identification of two major susceptibility loci. PLoS Genet 2009; 5: e1000421.

2 Cho $\mathrm{MH}$, Boutaoui N, Klanderman BJ, et al. Variants in FAM13A are associated with chronic obstructive pulmonary disease. Nat Genet 2010; 42: 200-202.

3 Cho $\mathrm{MH}$, Castaldi PJ, Wan ES, et al. A genome-wide association study of COPD identifies a susceptibility locus on chromosome 19q13. Hum Mol Genet 2012; 21: 947-957.

4 Wilk JB, Shrine NR, Loehr LR, et al. Genome-wide association studies identify CHRNA5/3 and HTR4 in the development of airflow obstruction. Am J Respir Crit Care Med 2012; 186: 622-632.

5 Cho $\mathrm{MH}, \mathrm{McD}$ onald ML, Zhou X, et al. Risk loci for chronic obstructive pulmonary disease: a genome-wide association study and meta-analysis. Lancet Respir Med 2014; 2: 214-225.

6 Wain LV, Shrine N, Miller S, et al. Novel insights into the genetics of smoking behaviour, lung function, and chronic obstructive pulmonary disease (UK BiLEVE): a genetic association study in UK Biobank. Lancet Respir Med 2015; 3: 769-781.

7 Hobbs BD, Parker MM, Chen H, et al. Exome array analysis identifies a common variant in IL27 associated with chronic obstructive pulmonary disease. Am J Respir Crit Care Med 2016; 194: 48-57.

8 Jackson VE, Ntalla I, Sayers I, et al. Exome-wide analysis of rare coding variation identifies novel associations with COPD and airflow limitation in MOCS3, IFIT3 and SERPINA12. Thorax 2016; 71: 501-509.

9 Hobbs BD, de Jong K, Lamontagne M, et al. Genetic loci associated with chronic obstructive pulmonary disease overlap with loci for lung function and pulmonary fibrosis. Nat Genet 2017; 49: 426-432.

10 Hancock DB, Eijgelsheim M, Wilk JB, et al. Meta-analyses of genome-wide association studies identify multiple loci associated with pulmonary function. Nat Genet 2010; 42: 45-52.

11 Repapi E, Sayers I, Wain LV, et al. Genome-wide association study identifies five loci associated with lung function. Nat Genet 2010; 42: 36-44.

12 Soler Artigas M, Loth DW, Wain LV, et al. Genome-wide association and large-scale follow up identifies 16 new loci influencing lung function. Nat Genet 2011; 43: 1082-1090.

13 Soler Artigas M, Wain LV, Miller S, et al. Sixteen new lung function signals identified through 1000 Genomes Project reference panel imputation. Nat Commun 2015; 6: 8658.

14 Wain LV, Shrine N, Artigas MS, et al. Genome-wide association analyses for lung function and chronic obstructive pulmonary disease identify new loci and potential druggable targets. Nat Genet 2017; 49: 416-425.

15 Wyss $\mathrm{AB}$, Sofer T, Lee MK, et al. Multiethnic meta-analysis identifies new loci for pulmonary function. bioRxiv 2017; preprint [https://doi.org/10.1101/196048].

16 Shrine N, Guyatt AL, Erzurumluoglu AM, et al. New genetic signals for lung function highlight pathways and pleiotropy, and chronic obstructive pulmonary disease associations across multiple ancestries. bioRxiv 2018; preprint [https://doi.org/10.1101/343293].

17 Busch R, Hobbs BD, Zhou J, et al. Genetic association and risk scores in a chronic obstructive pulmonary disease meta-analysis of 16,707 subjects. Am J Respir Cell Mol Biol 2017; 57: 35-46.

18 Castaldi PJ, Dy J, Ross J, et al. Cluster analysis in the COPDGene study identifies subtypes of smokers with distinct patterns of airway disease and emphysema. Thorax 2014; 69: 416-423.

19 Sakornsakolpat P, Prokopenko D, Lamontagne M, et al. Expanded genetic landscape of chronic obstructive pulmonary disease reveals heterogeneous cell type and phenotype associations. bioRxiv 2018; preprint [https://doi. org/10.1101/355644].

20 Manichaikul A, Hoffman EA, Smolonska J, et al. Genome-wide study of percent emphysema on computed tomography in the general population. The Multi-Ethnic Study of Atherosclerosis Lung/SNP Health Association Resource Study. Am J Respir Crit Care Med 2014; 189: 408-418.

21 Cho $\mathrm{MH}$, Castaldi PJ, Hersh CP, et al. A genome-wide association study of emphysema and airway quantitative imaging phenotypes. Am J Respir Crit Care Med 2015; 192: 559-569.

22 Castaldi PJ, Cho MH, San Jose Estepar R, et al. Genome-wide association identifies regulatory Loci associated with distinct local histogram emphysema patterns. Am J Respir Crit Care Med 2014; 190: 399-409.

23 Boueiz A, Lutz SM, Cho MH, et al. Genome-wide association study of the genetic determinants of emphysema distribution. Am J Respir Crit Care Med 2017; 195: 757-771.

24 McDonald ML, Cho MH, Sorheim IC, et al. Common genetic variants associated with resting oxygenation in chronic obstructive pulmonary disease. Am J Respir Cell Mol Biol 2014; 51: 678-687.

25 Lee JH, Cho MH, Hersh CP, et al. IREB2 and GALC are associated with pulmonary artery enlargement in chronic obstructive pulmonary disease. Am J Respir Cell Mol Biol 2015; 52: 365-376.

26 Lee JH, Cho MH, Hersh CP, et al. Genetic susceptibility for chronic bronchitis in chronic obstructive pulmonary disease. Respir Res 2014; 15: 113.

27 Hardin M, Cho M, McDonald ML, et al. The clinical and genetic features of COPD-asthma overlap syndrome. Eur Respir J 2014; 44: 341-350.

28 Terzikhan N, Sun F, Verhamme FM, et al. Heritability and genome-wide association study of diffusing capacity of the lung. Eur Respir J 2018; 52: 1800647.

29 Hallberg J, Iliadou A, Anderson M, et al. Genetic and environmental influence on lung function impairment in Swedish twins. Respir Res 2010; 11: 92.

30 Forster RE II. The single-breath carbon monoxide transfer test 25 years on: a reappraisal. 1-Physiological considerations. Thorax 1983; 38: 1-5.

31 Hughes JM, Pride NB. Examination of the carbon monoxide diffusing capacity (DLCO) in relation to its KCO and VA components. Am J Respir Crit Care Med 2012; 186: 132-139. 